\title{
Accuracy of Reinnervation of Rat Internal Intercostal Muscles by Their Own Segmental Nerves
}

\author{
V. J. Hardman and M. C. Brown \\ The University Laboratory of Physiology, Oxford OX13PT, England
}

\begin{abstract}
The positions of internal intercostal motoneurons within their motor pool were studied, following reinnervation of the intercostal muscles by their original nerves. Six to 9 weeks after proximal nerve section in 10-d-old and adult rats, 0.1 $\mu l$ injections of wheat germ agglutinin (WGA)-HRP were made in the distal part of the reinnervated internal intercostal muscle. The corresponding region of the contralateral control muscle was also injected. The positions of the retrogradely labeled motoneurons were mapped in $100 \mu \mathrm{m}$ transverse sections of thoracic spinal cord that had been stained for HRP according to the method of Mesulam (1982). In normal rats, motoneurons innervating distal muscle fibers are found largely in the more dorsal part of the internal intercostal motoneuron pool (Hardman and Brown, 1985). In adult rats, regenerated motor axons did not show any selectivity; distal muscle fibers were innervated by motoneurons whose cell bodies were distributed throughout the internal intercostal pool. However, in rats operated on at 10 d of age, distal intercostal muscle fibers were reinnervated by motoneurons that were distributed mainly in the dorsal part of the motor pool. These results support the view that positional signals may be of importance in organizing the distribution of axon terminals within muscles during development.
\end{abstract}

This paper is concerned with the way in which motor axons distribute their terminals within a muscle. It is clear that they are not distributed at random. Several studies have shown that there are consistent (albeit not very strong) correlations between the position of a motoneuron's cell body in the motor pool and the position in the muscle where its terminals make synapses. Most studies have related the rostrocaudal location of a motoneuron in the cord to the particular preferred location of its terminals in the muscle (Browne, 1950; Swett et al., 1970; Duron et al., 1979; Brown and Booth, 1983; Bennett and Lavidis, 1984). In the case of the internal intercostal muscles, however (Hardman and Brown, 1985), the organization in the cord is in the dorsoventral axis. Dorsally placed motoneurons tend to innervate the most distal part of the muscle (i.e., the region nearest to the sternum), and ventrally placed neurons innervate the proximal part of the muscle.

One way in which an ordered relationship between the po-

Received Apr. 21, 1986; revised July 22, 1986; acceptcd Scpt. 22, 1986.

V.J.H. is an M.R.C. Scholar. This work was supported by Action Research for the Crippled Child.

Correspondence should be addressed to M. C. Brown, The University Laboratory of Physiology, Parks Road, Oxford OX13PT, England.

Copyright (C) 1987 Society for Neuroscience $0270-6474 / 87 / 041031-06 \$ 02.00 / 0$ sition of a motoneuron soma in a motor pool and the location of its motor unit in a muscle could be established is through some form of chemoaffinity mechanism that acts during development either on the pathway within the muscle or at the neuromuscular junction. Alternatively, the relationship might arise simply through a sequential outgrowth of motor axons. If the latter were the case, then axons simultaneously reinnervating a muscle could never restore a normal innervation pattern. We set out to test this hypothesis in the internal intercostal muscles of rats by cutting the intercostal nerve proximally in the 5th, 6th or 7 th intercostal space in 10-d-old and adult rats. We found that motoneurons whose axons were cut in 10-d-old animals were able to restore an approximately normal intramuscular pattern of connections, while axons sectioned in adult rats were unable to do so. These results do not favor the view that ordered sequential outgrowth during development is necessary for the establishment of the intramuscular pattern of motor innervation.

\section{Materials and Methods}

Surgery. Successful results were obtained from 9 Wistar rats operated on at $10 \mathrm{~d}$ of age, and 7 Wistar rats operated on at 6-8 wceks of agc. The neonatal animals were anesthetized with ether and the adults with intraperitoneal injections of chloral hydrate $(1 \mathrm{ml} / 100 \mathrm{gm}$ of $3.5 \%$ solution). The left intercostal nerve of the 5 th, 6 th or 7 th intercostal space was exposed by teasing apart the muscle fibers of the overlying intercostal muscles at the lateral margin of the serratus dorsalis muscle, and then sectioned. A piece of $7 / 0$ black thread was sutured onto the external intercostal muscle to mark the operated rib space.

Motoneuron labeling. Six to 9 weeks after nerve section, the rats were anesthetized with chloral hydrate; the operated segment was identified by the marker thread. In several rats, the muscle in the operated rib space appeared atrophied when compared with neighboring muscles. The rib space was traced from the point of operation to the end of the external intercostal muscle, which is within $5 \mathrm{~mm}$ of the sternocostal junction. $\Lambda \mathrm{t}$ this point, two $0.1 \mu \mathrm{l}$ injections of $2 \%$ wheat germ agglutinin (WGA)-conjugated HRP (WGA-HRP; Sigma) in $0.9 \%$ saline containing $0.1 \%$ fast green were made into the distal part of the internal intercostal muscle. A pair of roughly symmetrical injections were made in the contralateral muscle as a control. In 3 additional rats the whole intercostal nerve was cut proximally and a pledget of gelfoam, soaked in $30 \%$ HRP, $2 \%$ lysolecithin solution, was placed on the cut end. These rats were used to gain an estimate of the total numbers and distribution of intercostal motoneurons.

Forty-eight hours later, the rats were deeply anesthetized with chloral hydrate and perfused with $100 \mathrm{ml}$ of heparin saline $(800 \mathrm{U} /$ liter in $0.9 \%$ saline), followed by $500 \mathrm{ml}$ of $1 \%$ paraformaldehyde $-1.25 \%$ glutaraldehyde in $0.1 \mathrm{M}$ phosphate buffer, $\mathrm{pH} 7.4$. The relevant portion of spinal cord was removed, postfixed for $1 \mathrm{hr}$ in the same fixative, and placed in $30 \%$ phosphate-buffered sucrose solution overnight. Sections of spinal cord of $100 \mu \mathrm{m}$ were cut on a freezing microtome and histochemically processed for HRP, according to the method of Mesulam (1982).

Measurement of motoneuron position. Motoneuron positions were measured as described in Hardman and Brown (1985). In brief, the vertical and horizontal coordinates of the labeled cell bodies were ex- 


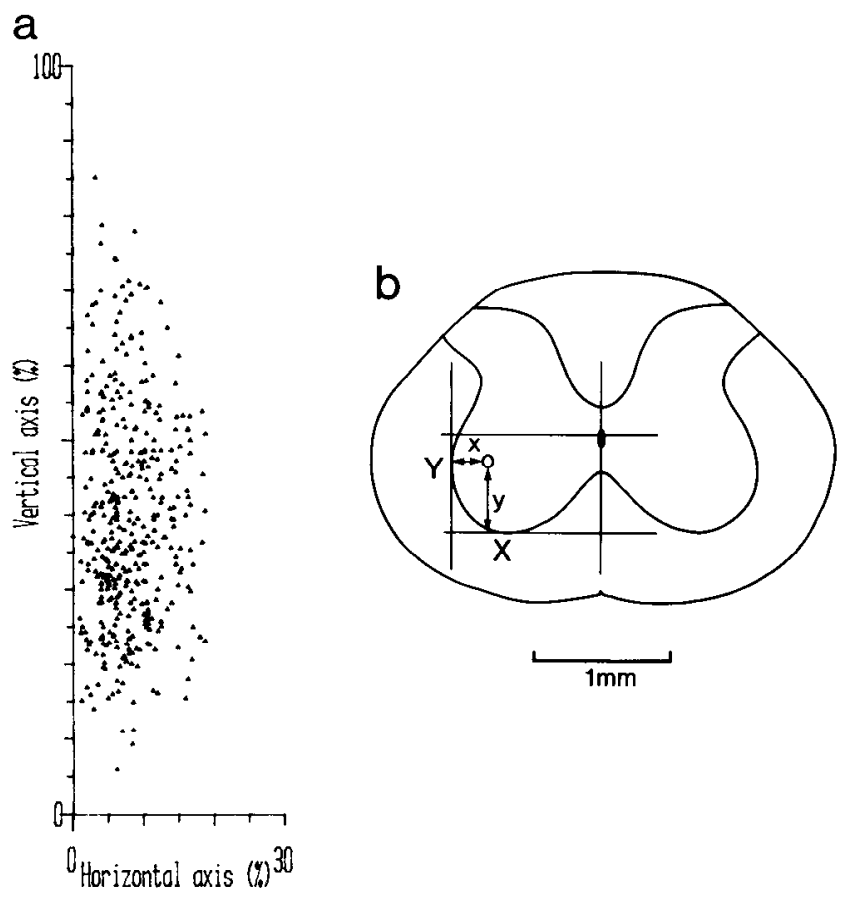

Figure 1. Distribution of internal intercostal motoneurons within the spinal cord, labeled by application of HRP to the whole sectioned intercostal nerve $(n=3)$. $a$, Scattergram illustrating the extent of the motor pool in the gray matter of the thoracic spinal cord. $b$, Diagram illustrating the method of normalizing motoneuron position.

pressed as percentages of the distances from the lateral and ventral margins of the gray matter to the central canal (see Fig. $1 b$ ). The image of the spinal cord sections was projected by camera lucida onto a Summagraphics digitizing pad and the position percentages calculated and stored on computer. Previous studies (Smith and Hollyday, 1983; Hardman and Brown, 1985) have shown that internal intercostal motoneurons are located close to the lateral margin of the ventral horn (less than $20 \%$, where $0 \%=$ the lateral margin of the gray matter and $100 \%=$ the center of the spinal cord; see Fig. 1). For the purposes of our experiments, any labeled motoneurons, whether experimental or control, with lateral positions greater than $20 \%$ were excluded from our results. It is likely that more medial motoneurons were labeled by the spread of HRP to the neighboring external intercostal muscle, although we cannot exclude the possibility that external intercostal motoneurons had reinnervated the experimental internal intercostal muscle. However, as the positions of external intercostal motoneurons are more restricted in the vertical axis, compared with internal intercostal motoneurons (Hardman and Brown, 1985), their inclusion would bias the overall distribution of cells in the vertical axis, and for this reason they were omitted. We have used the Mann-Whitney $U$ test to compare the experimental and control data (Seigel, 1956).

Electrophysiology. In 5 of the adult experiments and 4 of the animals denervated at $10 \mathrm{~d}$ of age, the sizes of motor units in the reinnervated muscle were sampled. The experimental side of the thoracic cage was dissected out immediately prior to perfusion fixation. The overlying musculature, including the external intercostal muscle, was dissected away, leaving the experimental internal intercostal muscle and its nerve, flanked by ribs and sternum. The preparation was pinned on the top of a Sylgard-lined dish and perfused with oxygenated Ringer's solution. The size of individual motor units was mapped by stimulating small filaments of intercostal nerve, using a suction pipette. These were stimulated at threshold levels to give all-or-none contractions of single units. The width of the contracting region was measured to the nearest halfmillimeter. This method was used by Dennis and Harris (1980). They compared its accuracy to that of mapping units by microelectrodes and found that the 2 methods gave similar results.

Muscle histochemistry. In 6 experiments (three adult and three $10 \mathrm{~d}$ ), the experimental intercostal muscle was frozen in liquid isopentane cooled by dry ice. Transverse sections, $20 \mu \mathrm{m}$, of the experimental and
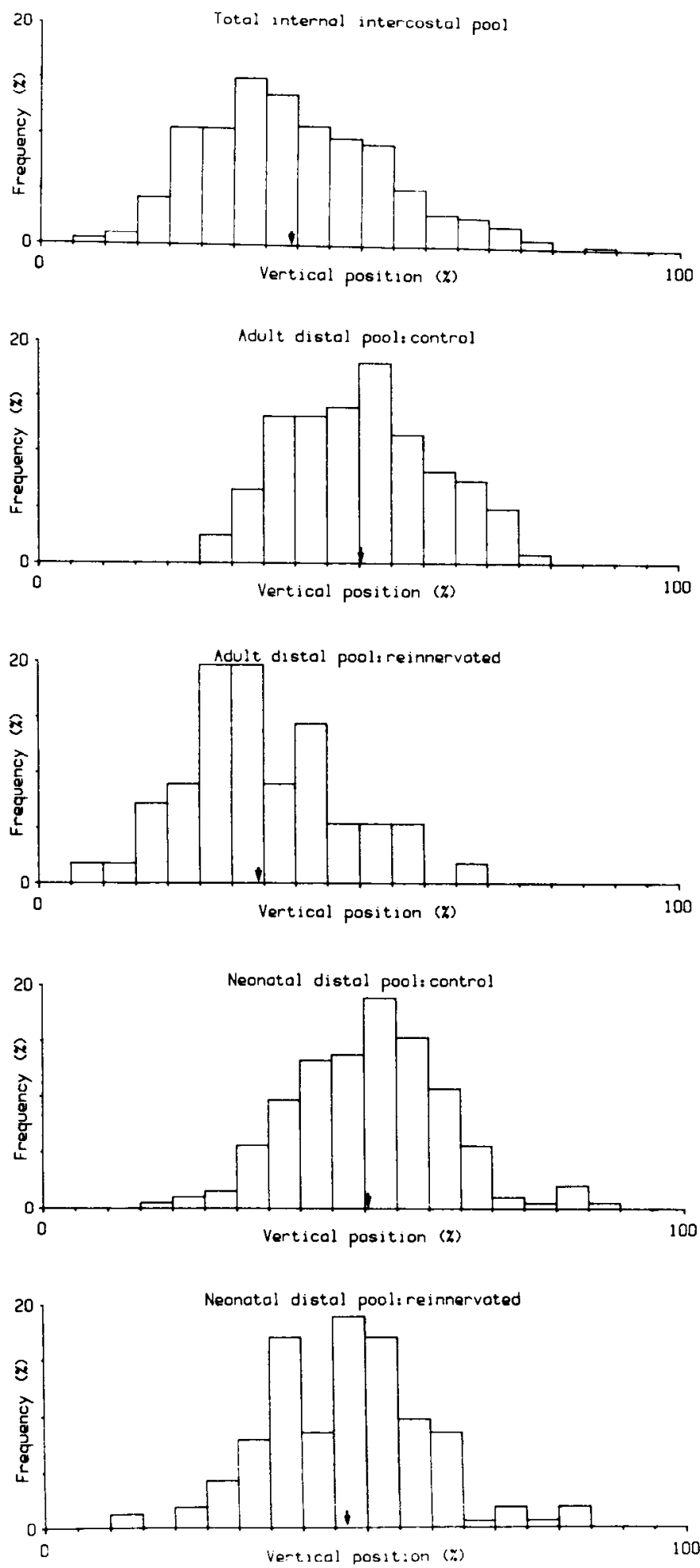

Figure 2. Histograms showing the relative positions in the vertical axis of the cord of motoneurons labeled following injections of HRP into internal intercostal muscles. From above down, the data are from the total labeling of the internal intercostal pool $(n=3 ; T=396)$; adult distal pool : control $(n-7 ; T=122)$; adult distal pool : reinnervated $(n=7 ; T=56)$; neonatal distal pool: control $(n=9 ; T=196)$; and the neonatal distal pool : reinnervated $(n=9 ; T=164)$. Arrows on the abscissa mark the mean values; $n=$ number of animals; $T=$ total number of cells labeled. 


\begin{tabular}{llll} 
& Group & $\begin{array}{l}\text { Number of labeled } \\
\text { cells (mean and } \\
95 \% \text { confidence } \\
\text { limits) }\end{array}$ & $\begin{array}{l}\text { Vertical } \\
\text { position } \\
\text { (mean and 95\% } \\
\text { confidence } \\
\text { limits) }\end{array}$ \\
\hline a. & Total normal internal intercostal nerve $(n=3)$ & $132.00 \pm 52.35$ & $39.32 \pm 1.41$ \\
b. & Adult distal pool : control $(n=7)$ & $17.43 \pm 11.32$ & $50.14 \pm 2.07$ \\
c. $\quad$ Adult distal pool : control $(n=5)$ & $19.00 \pm 9.66$ & $46.07 \pm 1.43$ \\
d. $\quad \quad$ Results from previous experiments & $8.00 \pm 4.35$ & $34.34 \pm 3.28$ \\
e. $\quad$ Neonatal distal pool : control $(n=9)$ & $22.11 \pm 8.78$ & $50.38 \pm 1.72$ \\
f. $\quad$ Neonatal distal pool : reinnervated $(n=9)$ & $18.44 \pm 13.02$ & $46.90 \pm 1.90$ \\
\hline
\end{tabular}

Significance of differences in the vertical position by Mann-Whitney $U$ test for large numbers: 1 . Adult reinnervated distal pool compared with (a) adult control distal pool $(Z=6.97, p<0.0001)$; (b) whole nerve $(Z=2.37,0.02>p>$ $0.01)$. 2. Neonatal reinnervated distal pool compared with (a) neonatal control distal pool $(Z=2.92,0.01>p>0.003)$; (b) whole nerve $(Z=6.20, p<0.0001)$. 3. Adult reinnervated distal pool compared with neonatal reinnervated distal pool $(Z=6.03, p<0.0001)$. 4. Neonatal control distal pool compared with adult control distal pool $(Z=0.34$; not significant).

of 2 control muscles were cut, using a cryostat. The sections were then histochemically stained for myosin ATPase according to the method of Brooke and Kaiser (1970). The relative numbers of muscle fibers, and the proportions of different fiber types, were calculated for samples taken from distal muscle regions in each case. These were compared with sections of normal muscles.

\section{Results}

\section{Normal distribution of motoneurons innervating internal} intercostal muscle

Figure $1 a$ shows the distribution of motoneurons labeled following application of HRP to the whole intercostal nerve. The cell bodies lie close to the lateral margin of the gray matter and are spread widely in the dorsoventral axis, with an interquartile range of $28.69-48.65 \%$. The distribution of motoneurons in the vertical axis is shown separately in the top histogram of Figure 2.

The position of the motoneurons on the vertical axis is related to the position of their axon terminals within the muscle. Dorsal motoneurons innervate distal muscle fibers, and ventral motoncurons innervate proximal muscle fibers. This topographical arrangement has been described for internal and external intercostal muscles (Hardman and Brown, 1985), and is confirmed in the results from control muscles in these experiments (Fig. 2-"Adult distal pool : control" and "Neonatal distal pool : control"). The interquartile ranges for the adult and neonatal control groups are $40.57-58.54 \%$ and $42.22-58.36 \%$, respectively.

The means, and their $95 \%$ confidence limits, for the vertical positions of motoneurons measured after (a) labeling of the whole nerve, (b and e) distal labeling, and (c) as taken from our previous data from distally labeled cells (Hardman and Brown, 1985) are given in Table 1.

\section{Projection pattern of intercostal motoneurons following reinnervation}

Adult

Histograms showing the distribution on the vertical axis of internal intercostal motoneurons labeled following distal injections in 7 adult rats are shown in Figure 2, "Adult distal pool : control" and "Adult distal pool : reinnervated." The average number of labeled cells was smaller on the reinnervated (left) side (see Table 1), and their positions in the motor pool did not correspond to those of contralateral (right) control cells. The interquartile range of the vertical position of reinnervating motoneurons was $26.92-42.50 \%$. This distribution is much closer to that of the whole internal intercostal motor pool (28.69$48.65 \%$ ) than that of neurons normally innervating distal muscle (40.57-58.54\%).

In addition to mapping the position of motoneuron somata with retrograde transport of HRP, we measured the sizes of 30 motor units from 5 reinnervated muscles to see if these differed from the values reported in the literature for normal muscles (Dennis and Harris, 1980). The mean width of units was 2.08 mm (see Fig. 3). This is in contrast to Dennis and Harris's (1980) figure of $1 \mathrm{~mm}$ for normal adult intercostal units, and to our results from neonatal reinnervated muscle (see below). Furthermore, 3 of the 30 units examined were greater than $5 \mathrm{~mm}$ in width (see also Dennis and Harris, 1980).

The distribution of muscle fiber types within the reinnervated intercostal muscles, as revealed by ATPase histochemistry, showed (Fig. 4) the typical fiber-type grouping patterns seen in previous reinnervation studics of other muscles (Karpati and Engel, 1968; Kugelberg et al., 1970). The variation in the number of fibers made it impossible to draw conclusions from such a small sample $(n=3)$, but the size of individual fibers and the total muscle thickness were in the normal range.

\section{Neonate}

The distribution of motoneurons innervating distal internal intercostal muscle following denervation at $10 \mathrm{~d}$ of age is shown in the histogram labeled "Neonatal distal pool : reinnervated" in Figure 2. It is positioned below the histogram for the unoperated side, labeled "Neonatal distal pool : control." The interquartile range for the vertical positions of the regenerated neonatal neurons was $38.35-53.85 \%$. The mean vertical position (see Table 1) was $46.90 \%$, which was similar to the figures for control injections in the present series $(50 \%)$ and in previous experiments $(46 \%)$.

A similar number of neurons was labeled after injection of experimental and control muscles. The mean size of motor units was approximately $1 \mathrm{~mm}$, and no abnormally large units were observed. The distribution of motor unit sizes was significantly different from that of regenerated adult units (Mann-Whitney $U$ test, $p=0.0075$ ). 


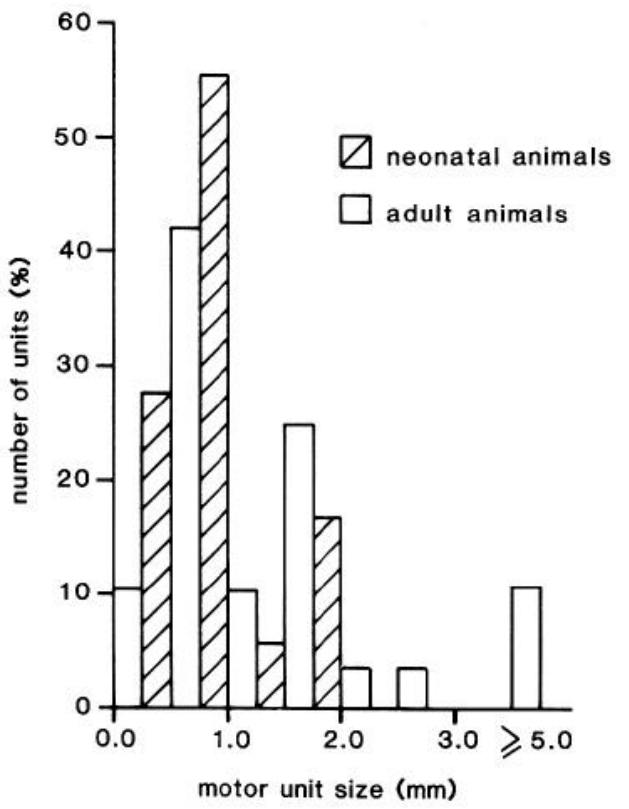

Figure 3. Histogram showing the distribution of motor unit sizes in adult and neonatally reinnervated internal intercostal muscles. Adults: $n=5$; number of units $=30$. Ten day sectioned group: $n=4$, number of units $=19$.

a

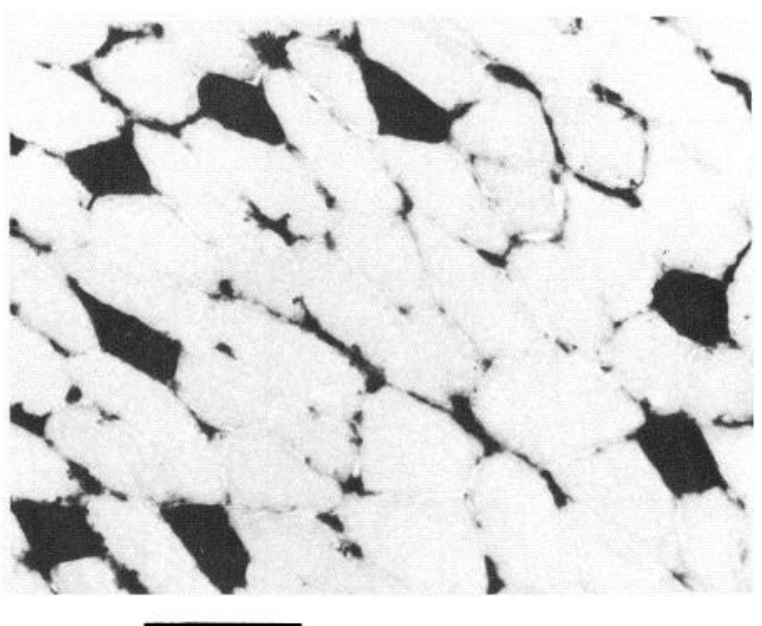

C

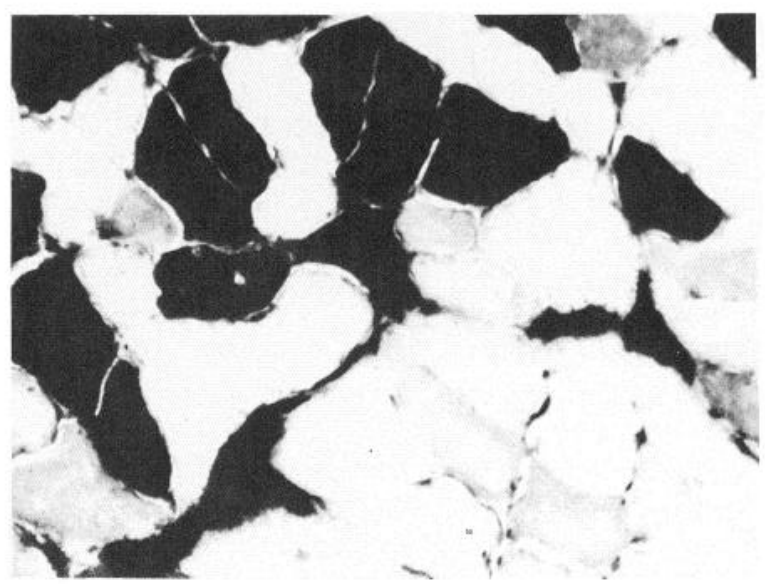

The number of muscle fibers was consistently lower than that in adult reinnervated and control muscles, but highly variable between individuals. ATPase histochemistry revealed fiber-type grouping within the reinnervated muscles (see Fig. 4). This demonstrates that, on reinnervation, neonatal motoneurons do not show muscle fiber-type specificity. This is in contrast to the spatial preference we have demonstrated in this study. The muscles contained a proportion of small uninnervated or regenerated fibers (see Fig. $4 d$ ). However, this feature was not studied in detail (see Wigston and Sanes, 1985).

Nerve section at $10 \mathrm{~d}$ might lead to death of motoneurons lying in the more ventral part of the internal intercostal pool. This would leave only more dorsally placed motoneurons, which could then reinnervate the whole muscle. Such differential death could, then, account for our results. This possibility was examined in 1 rat, whose intercostal nerve had been cut at $10 \mathrm{~d}$. We applied an HRP-soaked pledget of gel foam to the whole intercostal nerve 4 weeks after the original operation. This labeled 96 internal intercostal motoneurons, suggesting that death of motoneurons did not have a major effect. In addition, the population of labeled cells had a distribution similar to the total pool data from normal adult rats, illustrated at the top of Figure 2 . The mean position of cells in the vertical axis was $34 \%$. Thus, differential motoneuron death cannot account for our results.
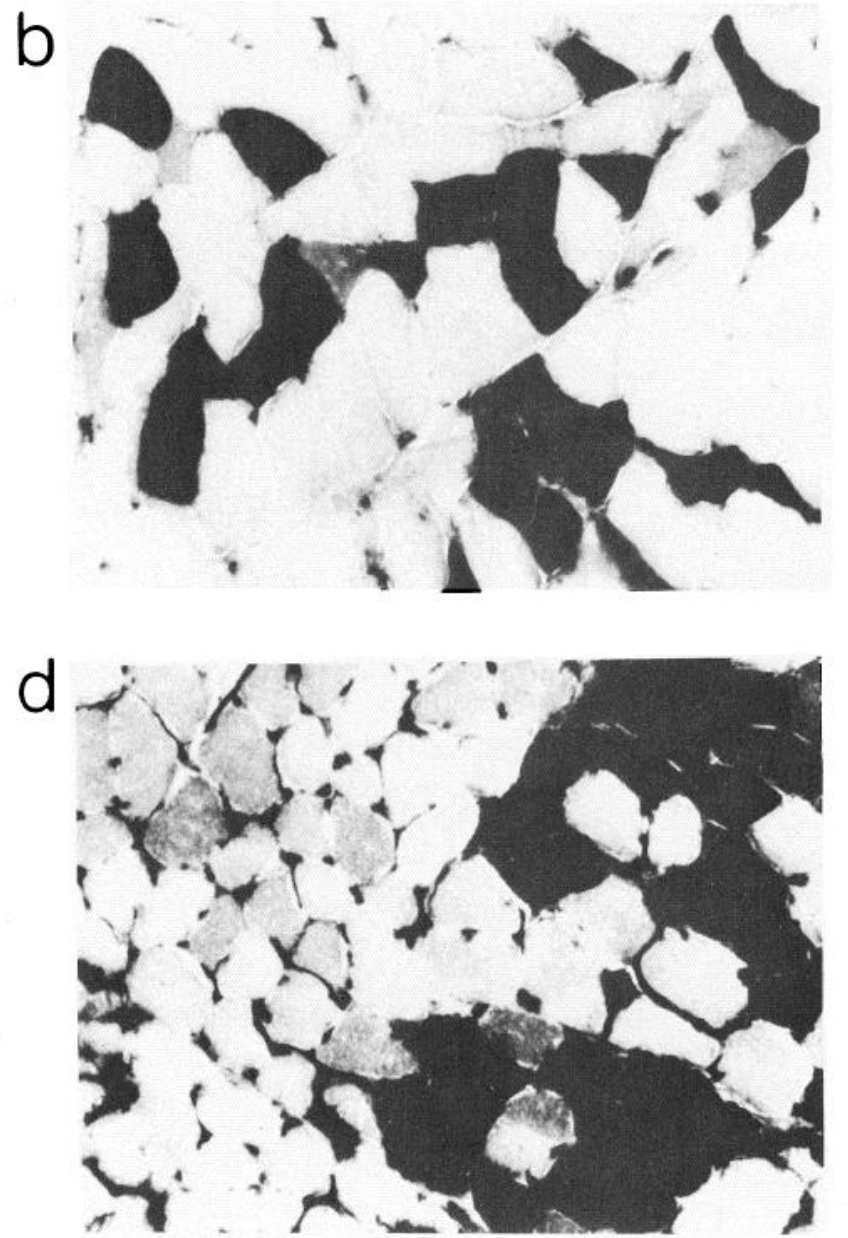

Figure 4. Micrographs of $20 \mu \mathrm{m}$ sections of distal internal intercostal muscle, stained for myosin ATPase, pH 4.2. Scale bar, $100 \mu \mathrm{m}$. $a$, Normal adult muscle. $b$, Adult reinnervated muscle. $c$ and $d$, Neonatally reinnervated muscles. 


\section{Discussion}

We have found a difference between the abilities of neonatal and adult internal intercostal motoneurons to reestablish the normal relationship between the position of a motoneuron in the spinal cord and the site in the muscle where its motor axon has terminal branches. After intercostal nerve regeneration, the adult motoneurons labeled by injections into the distal part of the muscle were not predominantly located in the dorsal part of the intercostal cord pool, as in normal animals. The positions of the labeled cells were statistically very different $(p<0.0001)$ from that of the controls and much more like those of the whole internal intercostal pool $(p<0.02)$ (see foot of Table 1).

Reinnervation in the neonate, however, established a more normal relationship, as can be seen by comparing the bottom 2 histograms of Figure 2. The data for distally labeled motoneurons in normal and neonatally reinnervated muscles do, however, differ significantly $(p<0.01)$ so that reinnervation accuracy was not perfect. However, it is certain that the motoneurons that do reinnervate the distal part of the internal intercostal muscle in neonates are not drawn at random from the total internal pool, for the data in the bottom and top histograms of Figure 2 are highly significantly different $(p<0.0001)$. In the neonate, too, the number of neurons labeled following reinnervation was the same as on the control side and the sizes of the motor units were also the same as those reported by Dennis and Harris (1980) in normal rats. Accuracy of reinnervation following nerve section has recently been reported for the facial nerve of the neonatal rat by Aldskogius and Thomander (1986). In their experiments, the individual muscles supplied by the nerve became reconnected with the right part of the facial nerve nucleus.

It therefore appears that the mechanisms used by outgrowing motor axons to establish the positions of their terminals within a muscle are still present (at least to some extent) in neonatal rats. Furthermore, one mechanism that is not essential for this organization is a timed sequence of axonal outgrowth. As the motor axons were cut and not crushed, it seems unlikely that the axons achieved their correct distribution simply by entering into their own old endoneurial sheaths [although Politis (1985) has reported that axons can show a preference for their own axonal stump when offered a choice between it and a foreign one]. It seems more probable that whatever chemical matching process is involved occurs at the synaptic target.

It is unclear at present how the postulated intramuscular cues relate to other reported examples of chemospecificity in the peripheral nervous system. These have mostly been concerned with rostrocaudal differences or with differences between various muscles. Wigston and Sancs (1985) have demonstrated that sympathetic preganglionic nerves show, even in the adult, a slight preference for intercostal muscle fibers taken from a rostrocaudal segment matching their own. On the whole, though, motoneurons in adult mammals have been found to be poor discriminators between their own and foreign muscles (Bernstein and Guth, 1961; Gillespie et al., 1983). Neonatal mammalian motoneurons, however, do show some selectivity in their ability to form synapses in different muscles (Gerding et al., 1977). In contrast with those of adult mammals, adult urodele motor axons show a strong preference for their own muscle (Grimm, 1971). Anuran motoneurons, too, can show a marked ability to find their correct muscles when sectioned at very early stages (Farel and Bemelmans, 1985) and can show some selec- tivity during reinnervation in the adult (Sayers and Tonge, 1982).

The failure of adult nerves to successfully reproduce the normal pattern of innervation here, even though the cut axons were closely apposed to the denervated muscle, could have several explanations. First, the postulated spatial signals may no longer be present in adult muscle. Second, even if the signals are present, selectivity could be lost for several reasons: (1) Motoneurons may fail to respond to any signal present after the period of synapse elimination (Brown and Booth, 1983; Bennett and Lavidis, 1984). (2) With maturity, motoneurons develop the ability to innervate any denervated or inactive muscle fibers (Brown et al., 1981; Sheard et al., 1984), and this might override any positional signal present. (3) As mentioned above, once regenerating motor axons enter a particular endoneurial tube in the distal stump of the nerve, their growth is constrained to that pathway. (4) The larger size of the adult intercostal muscle may make it more difficult for motoneurons to sample different muscle regions. This hypothesis is supported by the observation that fewer adult motoneurons reinnervate distal muscle (see Table 1).

Our results on motor unit size are similar to the findings of Dennis and Harris (1980) (see also Sheard et al., 1984), who also studied the reinnervation of rat intercostal muscles. They found that following neonatal axotomy, regenerated motor units were of normal size, suggesting either selective reinnervation or an inability to form larger units at that stage. We found that adult regenerated motor units were more variable in size although, unlike Dennis and Harris (1980), we did not find that all motor units were enlarged. This may have been due to the longer recovery time ( 9 weeks) used in this study, as compared with their interval of 2-8 weeks. It is important to distinguish between the area over which the muscle fibers of a motor unit are distributed in a muscle, which we and Dennis and Harris (1980) measured, and the number of muscle fibers in a unit that seems to be normal in reinnervated muscle (Gordon and Stein, 1982).

In agreement with reinnervation studies that have examined fiber-type arrangements in adults (Karpati and Engel, 1968; Kugelberg et al., 1970) and neonates (Soileau and Thompson, 1985), we did not find any reestablishment of normal fiber-lype distributions. In neonates, this may have been related to the fact that synapse elimination does not seem to contribute to the formation of motor units of uniform fiber type (Thompson et al., 1984), as it does to the formation of units of ordered spatial distribution (Brown and Booth, 1983; Bennett and Lavidis, 1984).

\section{References}

Aldskogius, H., and L. Thomander (1986) Selective reinnervation of somatotopically appropriate muscles after facial nerve transection and regeneration in neonatal rats. Brain Res. 375: 126-134.

Bennett, M. R., and N. A. Lavidis (1984) Development of the topographical projection of motor neurons to a rat muscle accompanies loss of polyneuronal innervation. J. Neurosci. 4: 2204-2212.

Bernstein, J. J., and L. Guth (1961) Nonselectivity in the establishment of neuromuscular connections following nerve regeneration in the rat. Exp. Neurol. 4: 262-275.

Brooke, M. H., and K. K. Kaiser (1970) Three "myosin adenosine triphosphatase" systems: The nature of their $\mathrm{pH}$ lability and sulphydryl dependence. J. Histochem. Cytochem. 18: 670-672.

Brown, M. C., and C. M. Booth (1983) Postnatal development of the adult pattern of motor axon distribution in rat muscle. Nature 304 : 741-742.

Brown, M. C., R. L. Holland, and W. G. Hopkins (1981) Motor nerve sprouting. Annu. Rev. Neurosci. 4: 17-42. 
Browne, K. M. (1950) The spatial distribution of segmental nerves to striate musculature of the hindlimb of the rat. J. Comp. Neurol. 93: 441-455.

Dennis, M. J., and A. J. Harris (1980) Transient inability of neonatal rat motoneurons to reinnervate muscle. Dev. Biol. 74: 173-183.

Duron, B., D. Marlot, and J. M. Macron (1979) Segmental motor innervation of the cat diaphragm. Neurosci. Lett. 15:93-96.

Farel, P. B., and S. E. Bemelmans (1985) Specific regeneration of developing spinal motoneurons in the absence of peripheral sensory fibres. Soc. Neurosci. Abstr. 11: 975.

Gerding, R., N. Robbins, and I. Antosiak (1977) Efficiency of reinnervation of neonatal rat muscle by original and foreign nerves. Dev. Biol. 61: 177-183.

Gillespie, M. J., T. Gordon, and P. R. Murphey (1983) Random reinnervation of $\mathrm{LG}$ and SOL muscles by their common nerve? Soc. Neurosci. Abstr. 9: 11.

Gordon, T., and R. B. Stein (1982) Time course and extent of recovery in reinnervated motor units of cat triceps surae muscles. J. Physiol. (Lond.) 323: 307-323.

Grimm, L. M. (1971) An evaluation of myotypic respecification in axolotls. J. Exp. Zool. 178: 479-496.

Hardman, V. J., and M. C. Brown (1985) Spatial organisation within rat motoneuron pools. Neurosci. Lett. 60: 325-329.

Karpati, G., and W. K. Engel (1968) "Type grouping" in skeletal muscles after experimental reinnervation. Neurology (Minneapolis) 18: 447 .

Kugelberg, E., L. Edstrom, and M. Abbruzzese (1970) Mapping of motor units in experimentally reinnervated rat muscle. Interpretation of histochemical and atrophic fibre patterns in neurogenic lesions. $J$ Neurol. Neurosurg. Psychiatry 33: 319-329.

Mesulam, M.-M. (1982) Tracing Neuronal Connections with Horseradish Peroxidase, pp. 127-131, Wiley, NY.

Politis, M. J. (1985) Specificity in mammalian peripheral nerve regeneration at the level of the nerve trunk. Brain Res. 328: 271-276.

Sayers, H., and D. A. Tonge (1982) Differences between foreign and original innervation of skeletal muscle in the frog. J. Physiol. (Lond.) 330: $57-68$.

Seigel, S. (1956) Nonparametric Statistics, pp. 120-127, McGraw-Hill, Kogakusha, Tokyo.

Sheard, P., C. D. McCaig, and A. J. Harris (1984) Critical periods in rat motoneuron development. Dev. Biol. 102: 21-31.

Smith, C. L., and M. Hollyday (1983) The development and postnatal organisation of motor nuclei in the rat thoracic spinal cord. J. Comp. Neurol. 220: 16-28.

Soileau, C., and W. J. Thompson (1985) Fibre type composition of single motor units in neonatally reinnervated rat soleus muscle. Soc. Neurosci. Abstr. 11: 101.

Swett, J. E., E. Eldred, and J. S. Buchwald (1970) Somatotopic cordto-muscle relations in efferent innervation of cat gastrocnemius. Am. J. Physiol. 219: 762-766.

Thompson, W. J., L. A. Sutton, and D. A. Riley (1984) Fibre type composition of single motor units during synapse elimination in neonatal rat soleus muscle. Nature 309: 709-711.

Wigston, D. J., and J. R. Sanes (1985) Selective reinnervation of intercostal muscles transplanted from different segmental levels to a common site. J. Neurosci. 5: 1208-1221 